\title{
EL POSITIVISMO DE KELSEN Y HART EN EL DERECHO INTERNACIONAL CONTEMPORÁNEO: UNA MIRADA CRÍTICA*
}

\author{
THE POSITIVISM OF KELSEN AND HART \\ IN CONTEMPORARY INTERNATIONAL \\ LAW: A CRITICAL PERSPECTIVE
}

Mario Iván Urueña-SÁnCheZ**

Fecha de recepción: 24 de junio de 2017

Fecha de aceptación: 15 de julio de 2017

Disponibilidad en línea: 30 de noviembre de 2017

\section{PARA CITAR este ARTículo / To CITE THIS ARTICle}

Urueña-Sánchez, Mario Iván, El positivismo de Kelsen y Hart en el derecho internacional contemporáneo: una mirada crítica, 31 International Law, Revista Colombiana de Derecho Internacional, 193-220 (2017). https://doi. org/10.11144/ Javeriana. il 15-31.pkhd

doi:10.11144/Javeriana.i115-31.pkhd

* Artículo producto de investigación en el marco del doctorado en derecho, Universidad del Rosario, Bogotá, Colombia.

** Doctorante en derecho, Universidad del Rosario. Estudios de doctorado en ciencia política, Universidad de Quebec, Montreal, Canadá. Magíster en geopolítica y seguridad global, Universidad de Roma La Sapienza, Italia. Politólogo, Universidad Nacional de Colombia. Profesor, universidades La Gran Colombia y Externado. Orcid ID: orcid.org/0000-0002-8040-6240. Correo electrónico: mario.uruena@urosario.edu.co 


\section{RESUMEN}

El presente artículo de investigación busca analizar los principales aspectos de las teorías del derecho internacional de Hans Kelsen y Herbert Hart desde una perspectiva crítica. Para lograr este objetivo, se realiza una investigación documental basada en un análisis de contenido de la obra directa de los autores, de otros autores que revalúan esta obra y de exponentes de la teoría crítica del derecho internacional y de las relaciones internacionales. Para el análisis propuesto, se escinden los planos epistemológico y normativo de las teorías citadas, lo que arroja como conclusión que hay una mayor sintonía entre las teorías positivistas y las críticas en lo que respecta al plano normativo mientras que en el epistemológico hay una fuerte discrepancia, en particular con el positivismo metodológico de Kelsen.

Palabras clave: Hans Kelsen; Herbert Hart; derecho internacional; teoría crítica; positivismo 


\section{ABSTRACT}

The present research article seeks to analyze the main aspects of Hans Kelsen and Herbert Hart's theories of international law from a critical perspective. To achieve this objective, a content analysis is carried out based on the documental research of the direct work of the authors, other authors who revalue this work and exponents of the critical theory of international law and international relations. For the proposed analysis, the epistemological and normative planes of the aforementioned theories are separated, which concludes that there is a greater harmony between the positivist and critical theories as far as the normative plane is concerned, while in the epistemological there is a strong discrepancy, particularly with Kelsen's methodological positivism.

Keywords: Hans Kelsen; Herbert Hart; international law; critical theory; positivism

\section{SUMARIO}

INTRODUCCIÓN.- I. LA EPISTEMOLOGÍA DEL DERECHO INTERNACIONAL DE KELSEN Y HART: PUNTOS DE VISTA Y PRESUPUESTOS.- II. LA NORMATIVIDAD DEL DERECHO INTERNACIONAL DE KELSEN y HART: DEL EUROCENTRISMO AL PACIFISMO internaCional.- Conclusión.- Bibliografía. 


\section{INTRODUCCIÓN}

¿Cómo entender críticamente la pertinencia de las teorías de Hans Kelsen y Herbert Hart para el derecho internacional contemporáneo? Esta pregunta surge de un interés por valorar la pertinencia de los autores que han servido de referencia académica preponderante para la teoría del derecho contemporáneo, en particular para el positivismo jurídico. Se parte del hecho de que ambos autores han sido de gran relevancia tanto para la teoría del derecho como para la constitución de regímenes legales estatales e internacionales. Su concepción del derecho ha dado pie a polémicas ligadas a la aplicación que se les da a sus teorías, ya sea por las lagunas que dejan sus obras como por los sesgos de interpretación entre quienes los han leído y han tomado decisiones políticas y jurídicas a partir de esta base.

Teniendo en cuenta que ambas teorías tienen una amplia variedad de temas de debate, este artículo se centrará en las consideraciones sobre el derecho internacional de las obras más significativas de cada autor: Teoría pura del derecho, de Kelsen y El concepto de derecho, de Hart.

Para leer estas obras en un contexto teórico más amplio, se ha elegido realizar una lectura crítica de ambos textos. Esta lectura es crítica en un doble sentido. Por una parte, en la revisión de teorías críticas ${ }^{1}$ pertinentes para el área de estudio (derecho internacional y relaciones internacionales). Aquí se definirán como teorías críticas aquellas cuyo "propósito es llegar claramente al foco de la perspectiva que fundamenta la teorización y su relación con otras perspectivas (para lograr una perspectiva de perspectivas) y de ahí abrir la posibilidad de escoger una perspectiva válida diferente desde la cual la problemática se convierta en una que sirva para crear un mundo alternativo"2.

1 Las teorías convocadas para este análisis son los critical legal international studies, el neogramscismo, el posestructuralismo, las sociologías históricas neomarxistas y neoweberianas, la teoría crítica y el constructivismo.

2 Robert W. Cox, Social Forces, States and World Orders: Beyond International Relations Theory, 10 Millennium: Journal of International Studies, 2, 126-155, 129 (1981). Disponible en: https://www.uni-erfurt.de/fileadmin/public-docs/Internationale_Beziehungen/cox.pdf 
Por el otro lado, se tomarán textos de estudiosos de la obra de Kelsen y Hart que han hecho el ejercicio de analizar y evaluar los conceptos primordiales de ambos autores en lo que compete al derecho internacional.

El análisis crítico que se va a hacer de ambas teorías va más allá de los medios por los cuales ambos autores llegan al conocimiento (epistemología). Debido a su importancia en los modelos de toma de decisión del derecho y en las implicaciones éticas que ello contrae, se ha adicionado un análisis normativo ${ }^{3}$ de ambos positivismos. Así, la idea central que pretende defender este texto apunta a que a pesar de que las teorías de Kelsen y Hart sobre el derecho internacional ameritan una valoración crítica debido a presupuestos epistemológicos derivados de su filiación con el positivismo metodológico y analítico, que de cierto modo terminan teniendo implicaciones éticas, su dimensión normativa amerita ser reivindicada al ser afín al cosmopolitismo y al pacifismo.

El reto que plantea revisar los planos epistemológico y normativo de Kelsen y de Hart es interesante, en la medida en que para las teorías críticas, epistemología y normatividad son codeterminantes. Es decir, la forma en que se comprende el mundo tiene una fuerte relación con lo que se prescribe de este. En otros positivismos, la división categórica entre sujeto y objeto y entre hechos y valores imposibilita esta codeterminación. Se verá cómo el positivismo jurídico en estas dos versiones se postula como una visión intermedia.

Este artículo se compone de dos partes. En la primera parte se realizará una discusión acerca de los elementos sobresalientes de la teoría del derecho internacional de Kelsen y Hart en lo correspondiente a su epistemología para de ahí hacer una valoración crítica de sus argumentos y de qué ideas alternativas plantean las perspectivas críticas. En la segunda parte se hace el mismo ejercicio, pero poniendo de presente los elementos normativos.

3 Para el presente artículo, normatividad se define como "(aquella) que trata no tanto con lo que es el campo de la teoría empírica, sino más bien con lo que podría o debería ser. De cómo el mundo debe ser ordenado, y de cuáles decisiones valorativas deben implementar los tomadores de decisión”. Comparar Paul R. Viotti \& Mark V. Kauppi, International Relations Theory, 16 (5 $5^{\text {a }}$ d., Pearson, Boston, 2012). 


\section{LA EPISTEMOLOGÍA DEL DERECHO INTERNACIONAL DE KELSEN Y HART: PUNTOS DE VISTA Y PRESUPUESTOS}

Tomando en cuenta que la epistemología "involucra las formas y los medios por los cuales llegamos a saber algo (o al menos lo que pensamos que sabemos) del mundo"4, este apartado del artículo analizará las teorías de Kelsen y Hart desde las visiones críticas a partir de: 1) el lugar desde el que se paran los autores para estructurar sus teorías, 2) la transformación del espacio en el que se desenvuelve cada nivel de análisis en estadios de la historia y 3) los fundacionalismos a los que recurren los autores para soportar su teoría.

En primer lugar, el "dónde" se paran los autores positivistas tiene una doble connotación. Por un lado, hace referencia al nivel de análisis desde el cual se observa la realidad mundial y por el otro lado, con las disciplinas involucradas en esa observación. Con relación a los niveles de análisis es justo aclarar que para efectos de este escrito se tomarán dos de ellos. El nivel estatal (municipal en Hart) será el primero de ellos, el cual hace alusión a la relación del Estado con sus asociados y el segundo nivel es el internacional, que parte del sistema internacional (o sociedad internacional) y su relación con los Estados (y de determinada manera con los individuos) $)^{5}$. Ante esta clasificación de niveles de análisis, lo primero que se puede decir de la epistemología de Kelsen y Hart es que hay una aparente contravía en el modo en el que ambos autores contemplan el derecho internacional. Mientras que la de Kelsen es una teoría cuya dirección de análisis va de lo internacional a lo interno, la teoría de Hart abre la puerta para un análisis "desde abajo" de la constitución del derecho internacional.

4 Paul R. Viotti \& Mark V. Kauppi, International Relations Theory, 6 (5a. ed., Pearson, Boston, 2012).

5 Para llegar a esta visión del derecho internacional fueron excluidas una perspectiva teórica y otra geográfica. Desde la teórica, se evitó tomar las teorías voluntaristas del derecho internacional, las cuales son profundamente rechazadas por Kelsen y Hart. Desde la perspectiva geográfica se dejaron a un lado los sistemas regionales de derecho internacional, puesto que estos no están incluidos en los debates propiamente teóricos del derecho internacional. 
En la teoría del derecho internacional, el autor austríaco plantea una postura monista con supremacía del derecho internacional radical. Es i) monista porque privilegia un sistema único en el que no hay contradicciones en las normas, ya que la penalización de un hecho ilícito no invalida la norma que lo prohíbe y por ende, no tienen cabida las posturas dualistas o pluralistas, ii) radical, porque no admite contradicción o coordinación entre sistemas normativos debido a que hay una determinación de una norma inferior por parte de una norma superior, lo que hace que la opción válida sea la de subordinación y iii) privilegia el derecho internacional puesto que lo equipara a la comunidad internacional que delimita los dominios de validez de los sistemas nacionales aun si el derecho internacional halla su aplicación en un momento posterior a la constitución de los sistemas nacionales ${ }^{6}$.

No obstante, el debate sobre la predilección de niveles de análisis en Kelsen es una cuestión que está lejos de haber sido zanjada. Este autor enmarca en su obra una palpable contradicción entre los principios neokantianos y la descripción positivista del derecho ${ }^{7}$. Esto trae consigo la necesidad de resolver una gran duplicidad del siglo XIX: la observación de todo orden coercitivo efectivo como Estado/Derecho ${ }^{8}$. Esta tensión neokantismopositivismo dificulta la tarea de etiquetar a Kelsen como alguien que ve el derecho estrictamente de "arriba hacia abajo". Pese a que el capítulo de Teoría pura del derecho dedicado al derecho internacional da la impresión de plasmar una visión fuertemente universalista inspirada en el filósofo alemán, buena parte de su obra pone el Estado como un ente preponderante, al que difícilmente se le puede superponer un poder que lo condicione ${ }^{9}$.

\footnotetext{
6 Hans Kelsen, Teoría pura del derecho (Universidad Nacional Autónoma de México, UNAM, México, 1982). Disponible en: enj.org/web/docman/doc_download/5191-teoria-pura-delderecho-hans-kelsen.html

7 Se puede considerar una aparente contradicción entre el neokantismo como enfoque del derecho de la vertiente iusnaturalista racional con el positivismo como un enfoque diferenciado.

8 José Antonio Sanz-Moreno, Kelsen y la unidad del Estado/Derecho: de las premisas kantianas a la ficción imaginada, 46 Política y Sociedad, 3, 175-189, 177 (2009). Disponible en: http:// revistas.ucm.es/index.php/POSO/article/view/POSO0909230175A/21807

9 Hans Kelsen, Teoría pura del derecho (Universidad Nacional Autónoma de México, UNAM,
} 
Por otra parte, en Hart se vislumbra una mayor coherencia para vindicar los niveles de análisis locales, cosa que induce a un análisis culturalista en el positivismo jurídico. Para llegar a este punto, el autor inglés compara dos tesis sobre el derecho internacional: la una es la de Kelsen en la que se considera que el derecho internacional, como todo derecho, proviene de una regla de reconocimiento, que le es superior. La otra es la de quienes señalan que el derecho internacional es un conjunto de reglas primarias de obligación que no están unidas por ninguna regla de reconocimiento. En esta disyuntiva, Hart coincide mayormente con los segundos pues afirma que la regla del reconocimiento no es condición necesaria para la existencia de reglas de obligación, lo que exime al derecho internacional de contener una regla básica o norma fundamental. Así, a diferencia de Kelsen y su tesis de unidad del sistema, Hart reconoce la existencia de distintas formas de estructura social ${ }^{10}$.

Sobre este punto de los niveles de análisis, las teorías críticas denuncian la pretensión universalista del positivismo kelseniano y tienen mayor sintonía con los postulados de Hart. Tal es el caso de los Critical Legal International Studies en los que autores como Anthony Carty se oponen al monismo, al supuesto universalismo y a la aparente neutralidad del derecho. La lógica que sustenta la postura de Carty se funda en que el elemento clave del derecho no es la sumisión, sino la identificación y la diferenciación. Para contraponerse al positivismo metodológico de Kelsen, Carty propone la teoría de la translación legal, la cual según sus palabras:

Debe comenzar en el entendimiento de la naturaleza parcial, multinivel y fragmentada de la sociedad internacional. Ella debe acercarse a esta realidad desde la base de las más novedosas percepciones traídas de la antropología legal y cultural. La sociedad internacional consiste, por encima de todo, en tradiciones culturales nacionales y regionales/continentales

10 Herbert Hart, The Concept of Law (Clarendon Press, Oxford, 1994). 
opuestas y autodiferenciadas, las cuales están entrecruzadas por sistemas comerciales y religiosos, que son más transnacionales ${ }^{11}$.

Aparte de la exhortación a observar las especificidades de la sociedad internacional, lo cual es una abierta crítica a la postura kelseniana, la teoría de la translación legal pone de manifiesto otros dos aspectos que fueron marginados por la visión del derecho de ambos autores. Por un lado, esta teoría se aleja del individualismo metodológico propio de la orientación epistemológica positivista al formular la vinculación de otras áreas del saber social — como la antropología - para acercarse a la complejidad del mundo social y sus distintas dimensiones. Por el otro lado, hay un interés de la teoría en mención por postular un tercer nivel de análisis jurídico. A los antes referidos niveles estatal e internacional, se puede vislumbrar cómo este último puede desdoblarse hacia el ámbito regional/continental, lo que de la misma manera involucraría otros campos de análisis como el cultural y el económico.

Huelga decir entonces que la teoría de Hart está a medio camino entre una teoría monista y metodológicamente individualista como la de Kelsen y una teoría de gran apertura epistemológica como los Critical Legal International Studies y su variante, la teoría de la translación legal. La "bisagra" hartiana se manifiesta al traer la dimensión doméstica como nivel de análisis determinante en la conceptualización del derecho, lo que matiza la posición "de arriba hacia abajo" del derecho de Kelsen, pero no incluye la óptica multinivel y fragmentada del enfoque crítico y, al hacer más plural el debate metodológico del derecho, trae el saber sociológico como campo de estudio de los tipos de regla.

En segundo lugar, las teorías de Kelsen y Hart ilustran un cuestionamiento que ha sido constante de las teorías críticas, en particular las posestructuralistas, a aquellas que tienen pretensión de cientificidad como lo son los diferentes positivismos.

11 Anthony Carty, Critical International Law: Recent Trends in the Theory of International Law, 2 The European Journal of International Law, EJIL, 1, $66-96$ (1991). Disponible en: http:// www.ejil.org/pdfs/2/1/2026.pdf 
Estas últimas suelen hacer un trastrocamiento de la relación espacio-tiempo. En el caso particular, se atestigua el paso del derecho estatal al derecho internacional (cambio geográfico de nivel de análisis) a una involución temporal (paso de derecho moderno a derecho primitivo), lo que desde la óptica crítica puede entenderse como la transformación del espacio en tiempo.

Esta transformación del espacio en tiempo parte por un lado de una idea central compartida por los dos autores y por el otro lado de un precepto que es más fuerte en Hart. En primera instancia, para Kelsen y Hart, la inexistencia de órganos y tribunales centralizados es un argumento relevante para identificar el derecho internacional como un derecho primitivo con respecto al derecho estatal. En el caso del pensador austríaco este desfase se basa en una especie de "ley evolutiva" de la humanidad, según la cual el orden jurídico que regula la convivencia entre los individuos es progresivo en la medida en que su grado de centralización va aumentando, cosa que en últimas favorece la pacificación del sistema jurídico mismo. De ahí que para él las 'comunidades primitivas' o las 'sociedades preestatales' tengan como característica primordial el hecho de contar con un orden jurídico descentralizado ${ }^{12}$. Este argumento es plenamente compartido con el pensador inglés, quien contempla del mismo modo la ausencia de centralización como un factor decisivo para distinguir entre el derecho estatal (municipal) y el internacional. Según él, la ausencia de una legislación internacional, de un sistema judicial y centralizado de sanciones imposibilita pensar en el derecho internacional como un sistema legal ${ }^{13}$.

A pesar de esta notable similitud, hay una discrepancia significativa en el papel que juega la violencia como elemento estructural de "lo moderno" entre los autores. Para Kelsen, la

12 Ramón Campderrich-Bravo, ¿Derecho internacional o guerra imperial? Hans Kelsen y Carl Schmitt ante la pacificación de las relaciones interestatales por medio del derecho, 43 Anales de la Cátedra Francisco Suárez, ACFS, 19-38, 24 (2009). Disponible en: http://revistaseug. ugr.es/index.php/acfs/article/view/816/940

13 Mehrdad Payandeh, The Concept of International Law in the Jurisprudence of H.L.A. Hart, 21 The European Journal of International Law, EJIL, 4, 967-995, 994 (2011). Disponible en: http://www.ejil.org/pdfs/21/4/2121.pdf 
recurrencia a la violencia es una constante tanto en el derecho estatal como en el derecho internacional, dado que en ambos órdenes jurídicos el Estado cumple una función eminentemente coactiva para crear obligación con su contraparte. Aunque en el derecho estatal hay órganos centralizados que hacen explícita esta función, en el derecho internacional la guerra y la represalia son las fórmulas de sanción preferidas ${ }^{14}$. Hart hace más amplio el desfase existente entre el derecho estatal y el internacional al discernir la frecuencia y la exhibición de la violencia entre ambos órdenes jurídicos. En el ámbito estatal, la violencia tiene una periodicidad más intensiva puesto que dispone de aparatos permanentes que pueden cumplir discretamente con este fin, mientras que en el internacional esta es pública. Además, en lo internacional hay tiempos largos de paz más extensos entre las guerras puesto que para los Estados el recurso a la violencia es costoso, conlleva riesgos y no siempre es concordante con sus necesidades ${ }^{15}$.

Un aspecto adicional que enuncia Hart para avivar la diferenciación entre las dicotomías estatal/moderno e internacional/ primitivo es el que tiene qué ver con las reglas secundarias de reconocimiento. Al partir de la pregunta ¿es el derecho internacional realmente derecho?, este autor señala que la inexistencia de reglas secundarias de reconocimiento hace que haya un mayor contraste entre los dos niveles del derecho. Como las reglas secundarias de reconocimiento son reglas cuyos destinatarios son los funcionarios y que suministran los criterios de validez de otras reglas en un sentido importante, se regresa al problema de la centralización e inexistencia de órganos de legislación internacional como indicadores del atraso temporal de los órdenes jurídicos ${ }^{16}$. Para Hart, ni siquiera el principio del pacta sunt servanda puede ser tomado como una regla de reconocimiento

14 Hans Kelsen, Teoría pura del derecho (Universidad Nacional Autónoma de México, UNAM, México, 1982).

15 Herbert Hart, The Concept of Law (Clarendon Press, Oxford, 1994).

16 Herbert Hart, The Concept of Law (Clarendon Press, Oxford, 1994). 
puesto que no todas las obligaciones internacionales surgen de los tratados y los acuerdos ${ }^{17}$.

No obstante, autores posteriores que han evaluado la obra de Hart critican fuertemente estas dicotomías e incluso llegan a disolverlas. Para Sebastián Green y Anthony H. Lesser, el derecho internacional no es un derecho primitivo y la clave para contravenir a Hart sobre este punto está en el artículo 38.1 del Estatuto de la Corte Internacional de Justicia que elabora la lista de las fuentes del derecho internacional. La referencia a este artículo por parte de los tomadores de decisión estatales ha sentado la base de una norma de reconocimiento consuetudinaria que ha derivado en el desarrollo progresivo del derecho internacional mediante el aprovisionamiento de estándares globalmente aceptados de comportamiento, de decisiones judiciales y de doctrina científica. El problema para ellos no está por lo tanto en que no haya una regla de reconocimiento como lo señala Hart, sino en que algunas de sus cláusulas no pueden ser fácilmente aplicadas con precisión ${ }^{18}$.

Puede apreciarse consecuentemente una pretensión compartida entre el positivismo metodológico de Kelsen y el analítico de Hart por hacer de una condición estrictamente geográfica algo que deviene en temporalidades divergentes. En Kelsen, la transformación del espacio en tiempo obedece más que todo a qué tan centralizado es un sistema de normas. En Hart, el derecho internacional se reduciría a un conjunto de normas por cuanto variables adicionales como la violencia o la carencia de reglas secundarias de reconocimiento forman un abismo entre las temporalidades de los dos derechos. El ahínco de Hart para discriminar el "adentro" del "afuera" termina haciendo su teoría más determinista en este punto. Este determinismo desconoce

17 Mehrdad Payandeh, The Concept of International Law in the Jurisprudence of H.L.A. Hart, 21 The European Journal of International Law, EJIL, 4, 967-995, 989 (2011).

18 Sebastián A. Green-Martínez, El derecho internacional actual ¿un derecho primitivo? Una reflexión a 50 años de "The Concept of Law", 61 Universidades, Unión de Universidades de América Latina y el Caribe, UDUAL, 51, 17-28, 19 (2011). Disponible en: http://www.redalyc. $\mathrm{org} / \mathrm{html} / 373 / 37322089003 /$. Anthony H. Lesser, H. L. A. Hart on International Law, 11 Kritikos, An International and Interdisciplinary Journal of Postmodern Cultural Sound, Text and Image, 1-25, 11 (2014). Disponible en: http://intertheory.org/HartandInternationalLaw-AHLesser.htm 
el carácter dinámico que puede tener un sistema para relativizar diferencias aparentemente hondas.

En tercer lugar, el énfasis que hacen los dos autores en separar tajantemente las esferas estatal e internacional exhibe desde el punto de vista de las teorías críticas dos fundacionalismos ${ }^{19}$ que vale la pena evaluar: i) la analogía entre derecho estatal e internacional y ii) la incuestionable existencia del Estado.

El primer fundacionalismo se desprende de la inquietud planteada en el punto anterior. Transformar la espacialidad estatal/internacional en la temporalidad primitivo/moderno invita en últimas a revaluar la analogía que hacen los autores entre las dos esferas del derecho. Cabe señalar que, aunque ambos autores toman como objeto de análisis central del derecho internacional, las semejanzas y discrepancias que tiene este con el derecho estatal, el propósito de esta analogía no es el mismo entre Kelsen y Hart. En consonancia con el punto anterior, Kelsen reclama una mayor afinidad entre ambos derechos a la vez que Hart identifica diferencias sustantivas. Por el lado de Kelsen, hay una asociación directa en lo que son las personas jurídicas en el derecho estatal ante el Estado y lo que son los Estados ante el derecho internacional ${ }^{20}$. Por el lado de Hart, la analogía entre derecho internacional y derecho municipal funciona en dos planos. Uno, dado en términos de contenido y que es procedente, en el que hay una identificación en cuanto a que ambos derechos están separados de la moral y otro, dado en términos de forma, en el que la analogía es improcedente y que fue antes mencionado: la inexistencia de reglas secundarias de reconocimiento. A pesar de que la analogía de Hart produce resultados combinados, el autor inglés marca una proximidad

19 Fundacionalismo puede ser definido como un enfoque general en relación con la epistemología que pretende como posible establecer bases incontrovertibles al conocimiento. Alexander MacLeod \& Dan O'Meara, Qu'est-ce qu'une théorie des relations internationales?, en Théories des relations internationales, contestations et résistances, 18-35 (Alexander MacLeod \& Dan O’Meara, Athéna, Outremont, Canada, 2010).

20 Hans Kelsen, Teoría pura del derecho (Universidad Nacional Autónoma de México, UNAM, México, 1982). 
entre ambos derechos cuando postula que no hay otro derecho más cercano al municipal que el internacional ${ }^{21}$.

Por su parte, desde la perspectiva crítica la analogía entre derecho estatal-internacional es problemática y puede llevar a un escenario de indefinición y ambigüedad, lo que se ve plasmado en tres problemas que allí se presentan. El primer problema es que el derecho estatal no es en sí mismo un concepto coherente y exento de polémica ${ }^{22}$. Pretender que todo orden jurídico, por la mera presencia formal de un Estado, presume la participación de órganos centralizados con capacidad de fijar obligaciones e impartir sanciones para dar validez a las normas es caer en un presupuesto cuyos efectos prácticos son, cuando menos, controvertibles. El segundo problema radica en la irreductibilidad de un derecho en el otro. En cada uno de los derechos, el tipo de actores involucrados, sus intereses y las metas buscadas varían considerablemente, lo que muestra un desafío importante a los marcos analíticos que, como los de Kelsen y Hart, buscan simplificar las dinámicas de ambas esferas. El tercer problema está en la presunción de asimilar el derecho estatal en el contexto exclusivo de los Estados constitucionales y de derecho. Concretamente en Hart, aunque su óptica trata de ser tan ecléctica como le es posible, se enfoca casi por completo en las ramas privada y penal del derecho ${ }^{23}$.

El segundo fundacionalismo en el que incurren los autores apunta a la figura misma del Estado y a la imperiosa necesidad de su existencia. Tanto en Kelsen como en Hart hay varias presunciones con respecto a lo que es el Estado: un Estado amoldado a la filosofía liberal europea y a todo lo que ello conlleva. Las teorías positivistas distan de pensar en estructuras estatales presentes a lo largo de la historia que puedan distanciarse de esa estrecha concepción. Una incertidumbre adicional aparece cuando se llega a considerar un escenario que cuestione con

21 Herbert Hart, The Concept of Law (Clarendon Press, Oxford, 1994).

22 Jason A. Beckett, The Hartian Tradition in International Law, 1 The Journal Jurisprudence, 51-83, 68 (2008). Disponible en: http://www.jurisprudence.com.au/vol1_beckett.pdf

23 Mehrdad Payandeh, The Concept of International Law in the Jurisprudence of H.L.A. Hart, 21 The European Journal of International Law, EJIL, 4, 967-995, 994 (2011). 
seriedad la potencial constitución de formas de organización jurídico-política alternativas al Estado.

Las teorías críticas disienten fuertemente del positivismo, al que acusan de ver al Estado como una entidad reificada, presocial y ahistórica. En primera instancia, lo señalan de naturalizar el Estado como un actor con una racionalidad que le es propia para imponer sanciones ${ }^{24}$. Al entender el Estado de esta manera se están dejando de lado 1) las motivaciones y percepciones de sus élites políticas y 2) la influencia que pueden ejercer actores transnacionales no estatales en el proceso de toma de decisión y en la firma de tratados internacionales.

En segunda instancia, el positivismo retrata al Estado como una entidad presocial y ahistórica. Esto quiere decir que lo ven como algo creado espontáneamente en un no tiempo ni lugar, como un algo dado y no como el producto de un proceso histórico y social específico. La implicación práctica de una idea tal se traduce en que se piense en el Estado como algo alejado de las relaciones de poder domésticas, algo parecido a un árbitro neutro cuyos intereses no se pueden identificar con los de sectores específicos de la sociedad. Ahí, teorías críticas como las sociologías históricas neomarxistas y neoweberianas brindan importantes luces para desmitificar al Estado. Este surge para ambas teorías en la interacción (lucha de clases u oposición de intereses) entre grupos o clases sociales en la Europa de los albores de la modernidad o incluso después. Además, que no solamente las formas de Estado surgen de factores domésticos, sino que también hay estructuras geopolíticas externas que lo condicionan y delimitan su soberanía ${ }^{25}$.

En resumen, para los estudiosos del derecho es pertinente valorar las teorías de Kelsen y Hart desde una perspectiva más amplia que dé cuenta del lugar desde el cual los autores se posan para explicar los fenómenos jurídicos internacionales, de

24 John Hobson, George Lawson \& Justin Rosenberg, Historical Sociology, en The International Studies Encyclopedia, 1-40 (Robert A. Denemark, ed., Wiley-Blackwell, London, 2010). Texto disponible en: http://eprints.lse.ac.uk/28016/1/Historical_sociology(LSERO.pdf

25 Hendrik Spruyt, Historical Sociology and Systems Theory in International Relations, 5 Review of International Political Economy, 2, 340-353, 346 (1998). 
los sesgos y el reduccionismo en el que ellos caen al momento de asociar sistemas jurídicos dispares y de las ideas prefijadas de las que ellos parten para dar coherencia interna a las ideas que defienden.

\section{LA NORMATIVIDAD DEL DERECHO INTERNACIONAL DE Kelsen y HART: DEL EUROCENTRISMO \\ AL PACIFISMO INTERNACIONAL}

A pesar de que la valoración crítica de las teorías positivistas de Kelsen y Hart en lo tocante a su epistemología descubre importantes falencias, con respecto a la normatividad de ambas teorías se debe afirmar que hay nociones muy importantes que vale la pena destacar de cara al derecho internacional actual. Acá, tres dimensiones se juzgan imperiosas para observar el positivismo metodológico y analítico: 1) su eurocentrismo, 2) la dicotomía entre imperialismo y pacifismo y 3 ) el desprendimiento de visiones coactivas del derecho internacional.

En primer lugar, el problema de los sistemas jurídicos al que se apegan los dos tipos de positivismo jurídico denota un cuestionamiento normativo más allá del epistemológico. Así, ante la pregunta jlos valores de quién?, es claro que, con matices, ambos autores terminan promoviendo un aparente universalismo que al final solo exalta valores liberales-occidentales. Si bien estos valores propenden por un humanitarismo democrático, este se supedita a lo que se entiende por humanitarismo y por derechos humanos desde la lectura de Occidente. El discurso de los derechos humanos es marcadamente eurocéntrico, ha sido consolidado y pulido desde el iusnaturalismo grecolatino y, salvo algunos aportes particulares, no ha requerido del concurso de cosmovisiones alternativas.

Aunque es evidente que Kelsen y en menor medida Hart caen en esa pretensión universalista, hay que establecer que ellos solo hacen parte de un espacio/tiempo determinado por la creencia auténtica de sus comunidades epistémicas por el punto arqui- 
médico. El punto arquimédico, como un punto de referencia fijo desde el cual se crea un sistema de conocimiento que lleve a la verdad, en el caso de los autores guarda relación con el eurocentrismo moderno. Tal punto arquimédico ha sido enérgicamente revaluado por las perspectivas críticas al pretender tomar una verdad relativa de alcance local y disfrazarla de universal, de tal manera que las poblaciones periféricas del mundo terminan por adoptar un sistema de valores exótico, que dista de tomar en consideración sus particularidades.

El eurocentrismo devenido en universalismo del positivismo metodológico tiene una implicación normativa igualmente sesgada: asociar la cosmovisión étnica con formas de gobierno universalizables. Como se ha referido antes, Kelsen y Hart privilegian una forma específica de organización jurídico-política llamada Estado; sobre la base de esa preexistencia del Estado, consideran que este se debe acomodar a los preceptos del Estado constitucional de derecho que en últimas está condicionado a la idea liberal de democracia. Sin embargo, esta democracia está alejada de la defensa del pluralismo que dice promover al no tomar en cuenta las singularidades de cada población. Con respecto al deber ser de la noción de democracia, Anthony Carty formula que esta no puede estar escindida de la etnicidad, puesto que "la democracia sin etnicidad carece de contenido y de dirección"26. Por su parte, el positivismo analítico está más cerca de esta postura al propender por los factores culturales y sociales cuando se habla de validar las reglas.

En segundo lugar, el positivismo de Kelsen no puede descalificarse por lo antes dicho. Tomando de la misma manera el contexto en el que vivió y elaboró su obra, su aspiración por privilegiar el derecho internacional sobre el derecho estatal con fines no solo metodológicos sino también éticos es digna de rescatar. Esto se consigue al defender normas universales que subsuman a las estatales y que eviten políticas expansionistas de un Estado

26 Anthony Carty, Myths of International Legal Order: Past and Present, 10 Cambridge Review of International Affairs, 2, 3-22, 13 (1997). 
sobre los demás. Su iniciativa de prescribir órdenes mundiales jurídicamente centralizados responde también al peligro que en su época representaron las ideologías imperialistas dominantes en Europa y a la necesidad de que la teoría pura del derecho sirviera para neutralizar tales proyectos y fuera garantía de la pacificación mundial ${ }^{27}$.

En la óptica de Kelsen, esta propuesta de pacificación se ve fortalecida por su legado kantiano. De ahí, el precepto que vale la pena exaltar es el del cosmopolitismo como superación del dogma de la soberanía. Este dogma está sustentado en la representación westfaliana de las relaciones internacionales, en la que la "razón de Estado" es la piedra angular cuya preponderancia direcciona el comportamiento estatal hacia sus semejantes. El problema de la representación westfaliana radica en la autopercepción que tienen las élites de un Estado como una unidad aislada y autárquica, lo que involucra una suerte de egoísmo estatal que le imposibilita lograr empatía con aquellos que están fuera de sus fronteras. Al final, esto hace factible recurrir a la agresión en contra de los demás Estados. Por su parte, el cosmopolitismo busca que los individuos que conforman la sociedad internacional se valgan de la moral para entablar relaciones de igualdad con sus pares y unirse en la defensa de causas afines a una ética universal $^{28}$. Kelsen se afirma como un pensador cosmopolita al reivindicar la primacía del derecho internacional como método para desmontar la noción tradicional de soberanía estatal y para restringir el uso de la fuerza por parte de los Estados ${ }^{29}$.

Las prescripciones de Kelsen hacia el pacifismo universal tienen también qué ver con la creación de instancias superiores en el derecho internacional que, en concordancia con su visión de la moral, tengan como centro de referencia el individuo por

27 Hans Kelsen, Teoría pura del derecho (Universidad Nacional Autónoma de México, UNAM, México, 1982).

28 Alexander MacLeod \& Dan O'Meara, Qu'est-ce qu'une théorie des relations internationales?, en Théories des relations internationales, contestations et résistances, 18-35 (Alexander MacLeod \& Dan O'Meara, Athéna, Outremont, Canada, 2010).

29 Ramón Campderrich-Bravo, ¿Derecho internacional o guerra imperial? Hans Kelsen y Carl Schmitt ante la pacificación de las relaciones interestatales por medio del derecho, 43 Anales de la Cátedra Francisco Suárez, ACFS, 19-38, 22 (2009). 
encima de los Estados. Para este efecto, el autor austríaco expone como su propuesta principal la creación de un tribunal internacional dotado de jurisdicción obligatoria que dé forma a una organización mundial para el mantenimiento de la paz y que tenga como guía la doctrina de la guerra justa al prohibir la guerra de agresión ${ }^{30}$.

Una innovación de este tribunal que termina siendo visionaria con respecto al derecho internacional contemporáneo es la de ampliar la responsabilidad penal, restringida exclusivamente a los Estados, para incluir a los individuos. Así, se combinan las formas objetivas y colectivas de responsabilidad con las subjetivas e individuales. El presupuesto para justificar esta ampliación de la responsabilidad se soporta en el apremio por prohibir la guerra como medio primitivo de sanción derivado de la acción estatal y entablar una responsabilidad individual concreta para aquellos que cometen hechos ilícitos que vayan contravía de la moral universal ${ }^{31}$.

Un poco menos enfática en este punto es la teoría de Hart, aunque en él también hay una clara prevención ante posturas revisionistas e incluso imperialistas de los Estados que pueden llevar a escenarios de confrontación interestatal. De ahí que el autor inglés plantee una doble comparación entre independencia con soberanía y autonomía. Volviendo a sus analogías entre derecho municipal y derecho internacional, Hart recuerda los peligros causados por la superposición de los individuos a las reglas. Del mismo modo ocurre con los Estados que en nombre de la soberanía deciden no ya protegerse de amenazas externas, sino que pretenden sojuzgar a otros más débiles. Por ello se hace vital limitar la soberanía como sinónimo de autonomía e

30 Ramón Campderrich-Bravo, ¿Derecho internacional o guerra imperial? Hans Kelsen y Carl Schmitt ante la pacificación de las relaciones interestatales por medio del derecho, 43 Anales de la Cátedra Francisco Suárez, ACFS, 19-38, 26 (2009).

31 José Antonio García-Saez, Guerra, coacción y autotutela en el pacifismo jurídico de Hans Kelsen, 11 Astrolabio, Revista Internacional de Filosofía, 177-189, 186 (2010). Disponible en: http://www.raco.cat/index.php/Astrolabio/article/view/239021/321279 
independencia mediante un conjunto de normas que impidan el recurso a la violencia ${ }^{32}$.

A pesar de que las teorías críticas de inspiración kantiana tienen un análisis similar al de la posición de Kelsen y afín a la de Hart, otras teorías críticas del derecho como el neogramscismo conminan a incluir un cuestionamiento al modo de producción económico como hecho determinante de la hegemonía actual. Esta, más que descansar en los Estados, descansa en un bloque histórico transnacional ${ }^{33}$. Esto genera dos repercusiones normativas dignas de ser tomadas en consideración por los estudiosos del derecho. Por un lado, el ejercicio de la opresión que sufren las capas mayoritarias de la sociedad internacional no tiene qué ver únicamente con el papel del Estado y del derecho, sino que allí se manifiesta la imposición de un sistema económico desigual en el cual se inserta el derecho. Infortunadamente, el derecho internacional ha sido instrumentalizado la mayor parte de las veces a favor del capital transnacional antes que al del bienestar de las poblaciones. No obstante, es imperativo entender el derecho también como una fuerza potencial de emancipación, mediante una contrahegemonía erigida "desde abajo" ${ }^{34}$

Por otro lado, la comunidad internacional debe reconocer otros actores más allá de los que reconocen las visiones positivistas, las cuales se limitan a hablar de Estados, individuos y derecho internacional. Hay que contemplar esta comunidad como algo que también compromete clases sociales que utilizan su ideología y su poder de coacción para subyugar a las masas marginadas. En caso contrario, la capacidad emancipadora del derecho internacional seguirá siendo inocua ante las relaciones de poder materiales del orbe.

32 Herbert Hart, The Concept of Law (Clarendon Press, Oxford, 1994).

33 A. Claire Cutler retoma la definición de bloque histórico de Antonio Gramsci para referirlo como "un conjunto complejo, contradictorio y discordante de relaciones sociales de producción (...) Un bloque histórico comprende una unidad entre naturaleza y espíritu (estructura y superestructura), unidad de opuestos y distintos". Comparar A. Claire Cutler, Gramsci, Law, and the Culture of Global Capitalism, 8 Critical Review of International Social and Political Philosophy, 4, 527-542, 538 (2005).

34 A. Claire Cutler, Gramsci, Law, and the Culture of Global Capitalism, 8 Critical Review of International Social and Political Philosophy, 4, 527-542, 538 (2005). 
En tercer lugar, si bien Kelsen promueve la primacía del derecho internacional y de la teoría pura del derecho como claves de la pacificación mundial, su convicción en el papel coactivo de la norma podría crear fisuras a la hora de lograr este fin. Es entonces cuando la teoría de Hart va un paso más allá al trascender la visión simplista del derecho internacional como "órdenes respaldadas por amenazas", lo que da pie a matizar la preponderancia de la guerra o de la represalia como maneras de establecer obligaciones para los Estados ${ }^{35}$. Acá, la intersubjetividad puede ser la clave hacia formas menos hostiles de conducción entre los individuos y los Estados. Para Kelsen, si bien hay una proposición de cambio en el ejercicio de la coacción de una forma estatal hacia una internacional, en ningún momento se pierde el rol que la violencia ocupa en el derecho ${ }^{36}$. Esto debido a que él establece que, así como el Estado tiene un carácter represivo, así mismo ocurre con el derecho internacional, ya que — como ha sido referido - este último encuentra en la guerra y la represalia sus formas de sanción. Incluso el tribunal internacional que él propone tendría que centralizar el ejercicio de la violencia para crear obligaciones. Para José Antonio García-Saez:

La guerra, entonces, se encuentra prohibida por el Derecho Internacional general precisamente porque es una sanción del Derecho Internacional (...) La eliminación de la guerra, por tanto, pasa por encontrar otro mecanismo que sea capaz de ejercer la coacción en el ámbito internacional (...) La construcción de ese mecanismo alternativo para Kelsen pasa inevitablemente por el establecimiento de un tribunal internacional ${ }^{37}$.

De su parte, Hart no hace la transferencia directa que sí hace Kelsen del papel de la violencia de la esfera estatal (municipal) a la internacional. Como se citó anteriormente, en el derecho municipal la violencia es esperada por su frecuencia, mientras que

35 Herbert Hart, The Concept of Law (Clarendon Press, Oxford, 1994).

36 Hans Kelsen, Teoría pura del derecho (Universidad Nacional Autónoma de México, UNAM, México, 1982).

37 José Antonio García-Saez, Guerra, coacción y autotutela en el pacifismo jurídico de Hans Kelsen, 11 Astrolabio, Revista Internacional de Filosofia, 177-189, 185-186 (2010). Disponible en: http://www.raco.cat/index.php/Astrolabio/article/view/239021/321279 
en el internacional esta es verdaderamente la ultima ratio para crear obligaciones. Para él, los sistemas legales son considerados independientemente del papel de la fuerza para desenvolverse como normas imperativas cuyo cumplimiento está sustentado en la presión social generalizada o incluso en el deseo de cumplimiento $^{38}$. La visión no coactiva del derecho internacional de Hart abre la puerta a una construcción social intersubjetiva de las reglas que se desprende tajantemente de un sistema represivo, lo que permitiría la reconstrucción de modos más cooperativos y democráticos de relación entre los pueblos. Acá hay una gran afinidad entre Hart y las teorías constructivistas.

En síntesis, en el complemento de las ideas de pacifismo internacional de Kelsen y de formas no coactivas de cumplimiento de las reglas de Hart, puede entreverse un interesante aporte del positivismo jurídico para construir regímenes jurídicos alternativos en el sistema internacional. Regímenes que tengan en cuenta la defensa efectiva de principios universales erigidos por el concurso y reconocimiento de la integridad de aquellos a quienes estas reglas obligan.

Finalmente, pese a las importantes contribuciones de las teorías de Kelsen y Hart a una posible construcción de mundos alternativos, para las teorías críticas estas prescripciones podrían parecer tímidas o estar a medio camino de cara a una transformación global. El paso de sujetos oprimidos a sujetos emancipados debe trascender el debate derecho/Estado o la discusión validez/eficacia para asentarse en la forma en que los individuos conciben el mundo y el lugar que ocupan en él. El reto es ir más allá de solo renunciar al recurso de la violencia para atribuirle al otro un reconocimiento pleno tanto en materia jurídica como material. Es cambiar los preceptos ideales sobre los cuales se instituyó el orden mundial para construir un mundo más equitativo y plural. Como lo esboza Paul Ricœur es

38 Jean d'Aspremont, Herbert Hart and the Enforcement of International Law: Substituting Social Disability to the Austinian Imperatival Handicap of the International Legal System (SSRN, Working Paper, 2012). Disponible en: https://papers.ssrn.com/sol3/papers.cfm?abstract_id=1995041 
"moverse de la desconfianza a la consideración y de la injusticia al respeto. Del miedo al respeto" 39 .

39 Anthony Carty, New Philosophical Foundations for International Law: From an Order of Fear to One of Respect, 19 Cambridge Review of International Affairs, 2, 311-330, 313 (2006). 
Conclusión

El anterior artículo tomó en consideración las teorías del positivismo metodológico de Hans Kelsen y el analítico de Herbert Hart para valorarlas desde teorías críticas del derecho internacional y las relaciones internacionales, además de la de autores que han evaluado críticamente su obra. Para ese fin se tuvieron en cuenta dos planos que junto a la ontología hacen parte de la integridad teórica interna: la epistemología y la normatividad. Para cada uno de estos planos se encontraron reflexiones dispares.

En lo concerniente al plano epistemológico, principalmente la teoría de Kelsen y de algún modo la de Hart no logran obtener una distancia remarcable con el positivismo científico de su tiempo. La pretensión de parsimonia que busca simplificar la realidad para hacerla inteligible y que es propia de la orientación epistemológica positivista atraviesa la obra de los dos autores estudiados. Entre las dimensiones que evidencian esta situación están la pretensión universalista, la metodología del punto arquimédico, las analogías y superposiciones de niveles de análisis, la transmutación del espacio en tiempo y los fundacionalismos de los que se parte sin la intención de cuestionamiento.

A pesar de que la observación epistemológica no representa una ruptura sustantiva con el universo positivista, en el plano normativo hay hallazgos muy interesantes que invitan a redescubrir la teoría de los dos autores estudiados. En el positivismo de David Hume, la pretensión de cientificidad hace que sus teorías no tengan por vocación prescribir escenarios alternativos, mucho menos si estos traen consigo un contenido moral o ético. Sin embargo, Kelsen y Hart tienen una normatividad explícita para hablar de un deber ser de la sociedad internacional, la cual puede entenderse como una normatividad abierta al cambio, que si bien no es transformadora ni revolucionaria sí abre la posibilidad de reformar el derecho internacional hacia un mundo más pacífico, en el que el recurso a la violencia sea superado. 
Es justamente en el plano de la normatividad en el que las teorías críticas hallan mayores puntos de confluencia con los positivismos de Kelsen y Hart. Entonces, en la normatividad, la opción de una metateoría jurídica puede ser vislumbrada para hacer del derecho un instrumento emancipador. 


\section{BIBLIOGRAFÍA}

Libros

Hart, Herbert, The Concept of Law (Clarendon Press, Oxford, 1994).

Kelsen, Hans, Teoría pura del derecho (Universidad Nacional Autónoma de México, UNAM, México, 1982). Disponible en: enj.org/web/docman/doc_download/5191teoria-pura-del-derecho-hans-kelsen.html

Viotti, Paul R. \& Kauppi, Mark V., International Relations Theory (5a ed., Pearson, Boston, 2012).

\section{Colaboración en obras colectivas}

Hobson, John; Lawson, George \& Rosenberg, Justin, Historical Sociology, en The International Studies Encyclopedia, 1-40 (Robert A. Denemark, ed., WileyBlackwell, London, 2010). Texto disponible en: http://eprints.lse.ac.uk/28016/1/ Historical_sociology(LSERO.pdf

MacLeod, Alexander \& O'Meara, Dan, Qu'est-ce qu'une théorie des relations internationales?, en Théories des relations internationales, contestations et résistances, 18-35 (Alexander MacLeod \& Dan O’Meara, Athéna, Outremont, Canada, 2010).

\section{Revistas}

Beckett, Jason A., The Hartian Tradition in International Law, 1 The Journal Jurisprudence, 51-83 (2008). Disponible en: http://www.jurisprudence.com.au/ vol1_beckett.pdf

Campderrich-Bravo, Ramón, ¿Derecho internacional o guerra imperial? Hans Kelsen y Carl Schmitt ante la pacificación de las relaciones interestatales por medio del derecho, 43 Anales de la Cátedra Francisco Suárez, ACFS, 19-38 (2009). Disponible en: http://revistaseug.ugr.es/index.php/acfs/article/view/816/940

Carty, Anthony, Critical International Law: Recent Trends in the Theory of International Law, 2 The European Journal of International Law, EJIL, 1, 66-96 (1991). Disponible en: http://www.ejil.org/pdfs/2/1/2026.pdf

Carty, Anthony, Myths of International Legal Order: Past and Present, 10 Cambridge Review of International Affairs, 2, 3-22 (1997).

Carty, Anthony, New Philosophical Foundations for International Law: From an Order of Fear to One of Respect, 19 Cambridge Review of International Affairs, 2, 311-330 (2006).

Cox, Robert W., Social Forces, States and World Orders: Beyond International Relations Theory, 10 Millennium: Journal of International Studies, 2, 126-155 (1981). Disponible en: https://www.uni-erfurt.de/fileadmin/public-docs/Internationale Beziehungen/cox.pdf 
Cutler, A. Claire, Gramsci, Law, and the Culture of Global Capitalism, 8 Critical Review of International Social and Political Philosophy, 4, 527-542 (2005).

García-Saez, José Antonio, Guerra, coacción y autotutela en el pacifismo jurídico de Hans Kelsen, 11 Astrolabio, Revista Internacional de Filosofía, 177-189 (2010). Disponible en: http://www.raco.cat/index.php/Astrolabio/article/view/239021/321279

Green-Martínez, Sebastián A., El derecho internacional actual ¿un derecho primitivo? Una reflexión a 50 años de "The Concept of Law", 61 Universidades, Unión de Universidades de América Latina y el Caribe, UDUAL, 51, 17-28 (2011). Disponible en: http://www.redalyc.org/html/373/37322089003/

Lesser, Anthony H., H. L. A. Hart on International Law, 11 Kritikos, An International and Interdisciplinary Journal of Postmodern Cultural Sound, Text and Image, 1-25 (2014). Disponible en: http://intertheory.org/HartandInternationalLaw-AHLesser. htm

Payandeh, Mehrdad, The Concept of International Law in the Jurisprudence of H.L.A. Hart, 21 The European Journal of International Law, EJIL, 4, 967-995 (2011). Disponible en: http://www.ejil.org/pdfs/21/4/2121.pdf

Sanz-Moreno, José Antonio, Kelsen y la unidad del Estado/Derecho: de las premisas kantianas a la ficción imaginada, 46 Política y Sociedad, 3, 175-189 (2009). Disponible en: http://revistas.ucm.es/index.php/POSO/article/view/ POSO0909230175A/21807

Spruyt, Hendrik, Historical Sociology and Systems Theory in International Relations, 5 Review of International Political Economy, 2, 340-353 (1998).

\section{Working paper}

D'Aspremont, Jean, Herbert Hart and the Enforcement of International Law: Substituting Social Disability to the Austinian Imperatival Handicap of the International Legal System (SSRN, Working Paper, 2012). Disponible en: https://papers.ssrn.com/ sol3/papers.cfm?abstract_id $=1995041$

\section{Tratados internacionales}

Organización de Naciones Unidas, Estatuto de la Corte Internacional de Justicia de la Organización de las Naciones Unidas, adoptado el 26 de junio de 1945. Disponible en: http://www.un.org/es/documents/icjstatute/ 
